\title{
Influences of the Perception of Self-Motion on Postural Parameters
}

\author{
E.A. KESHNER, P.T., Ed.D.,1,2 K. DOKKA, M.S.,1,3 and R.V. KENYON, Ph.D. 4
}

\begin{abstract}
We examined how spatial and temporal characteristics of the perception of self-motion, generated by constant velocity visual motion, was reflected in orientation of the head and whole body of young adults standing in a CAVE ${ }^{\circledR}$, a virtual environment that presents wide field of view stereo images with context and texture. Center of pressure responses from a force plate and perception of self-motion through orientation of a hand-held wand were recorded. The influence of the perception of self-motion on postural kinematics differed depending upon the plane and complexity of visual motion. Postural behaviors generated through the perception of self-motion appeared to contain a confluence of the cortically integrated visual and vestibular signals and of other somatosensory inputs. This would suggest that spatial representation during motion in the environment is modified by both ascending and descending controls. We infer from these data that motion of the visual surround can be used as a therapeutic tool to influence posture and spatial orientation, particularly in more visually sensitive individuals following central nervous system (CNS) impairment.
\end{abstract}

\section{INTRODUCTION}

$\mathbf{E}^{\mathrm{x}}$ XPOSURE TO WIDE FIELD OF VIEW visual motion produces an illusion of self-motion in the direction of the moving visual field. ${ }^{1}$ This visually induced perception of self-motion (vection) reflects an interaction between visual and vestibular information and is generated by visual inputs to the cortical vestibular centers. ${ }^{2}$ The optical flow pattern creates a compelling illusion of self-motion, but this illusion is not corroborated by inertial forces transmitted through the vestibular sense organs. ${ }^{3}$ Thus there is a mismatch between visual and vestibular feedback that has the potential to affect constancy of the perception of self-motion. Indeed, vection responses have been shown to have long time constants ${ }^{3}$ and fluctuating intensities. ${ }^{4}$ In this study, we have explored whether the temporal and spatial characteristics of the visually induced perception of self-motion are exhibited differentially in the postural kinematics of the head and in center of pressure by inducing a vection response in an immersive, stereo virtual environment (VE). We hypothesized that head orientation would directly reflect response direction and magnitudes necessary to compensate for the perceived vertical, but that to maintain a stable posture, center of pressure responses would be modulated by segmental inputs acting on the descending visual and vestibular signals.

1Sensory Motor Performance Program, Rehabilitation Institute of Chicago, Chicago, Illinois.

2Department of Physical Medicine and Rehabilitation, Feinberg School of Medicine, Northwestern University, Chicago, Illinois. ${ }^{3}$ Department of Bioengineering, University of Illinois at Chicago, Chicago, Illinois.

${ }^{4}$ Department of Computer Science, University of Illinois at Chicago, Chicago, Illinois. 


\section{METHODS}

Twelve healthy young adults (20-34 years) gave informed consent in accordance with the guidelines of the Institutional Review Board at the University of Illinois at Chicago. Subjects were immersed in a three-dimensional, complex texturemapped, stereoscopic visual scene generated by a VE called the CAVE ${ }^{\circledR}$ (CAVE Automatic Virtual Environment) that is composed of four screens arranged in a 10-ft cube with projections (Mirage 5000 projectors, Christie Digital Systems, Cypress, CA) on three walls and the floor. ${ }^{5}$ An SGI Onyx with two Infinite Reality graphics pipelines controlled the projected images. Image resolution was $1024 \times 1024$ pixels with a refresh rate of $120 \mathrm{~Hz}$ (an effective stereo refresh rate of $60 \mathrm{~Hz}$ ). Total display system latency from the time a subject moved to the time a new stereo image was displayed was 50-75 ms. Subjects wore stereo shutter glasses (Stereographics Corp., San Rafael, CA) which limited field of view to $100^{\circ}$ of horizontal binocular vision and $55^{\circ}$ in the vertical direction. Subjects stood on a force plate (Advanced Medical Technology Inc., Watertown, MA) which measured the ground reaction forces at a sampling frequency of $1000 \mathrm{~Hz}$. Two six degree-of-freedom tracking devices (Intersense, Bedford, MA), calibrated to an accuracy of $\pm 1.3 \mathrm{~cm}$, tracked head position and angular orientation of a hand-held wand.

\section{Scene characteristics}

The VE (scene) consisted of a room containing round columns with patterned rugs and a painted ceiling. ${ }^{6}$ Rug patterns were texture mapped on the floor. The subject stood in the center of the room between two rows of columns. Beyond the virtual room was a landscape consisting of mountains, meadows, sky, and clouds. The floor was the distance from the subject's eyes to the virtual floor and the nearest column was $4.6 \mathrm{~m}$ away. The visual experience presented to the subjects was that of being immersed in a volume filled with three dimensional objects at various distances from the subject. Head tracking insured that the virtual objects retain their true perspective and position in space regardless of the subjects' movement.

\section{Procedures}

Subjects stood on the force plate with their arms at their sides and feet together while exposed to four visual stimuli in random order: natural visual feedback with motion of the scene matched to motion of the head, pitch or roll motion of the scene at a constant velocity (36 degrees/sec), and combined pitch and roll motion of the scene. Combined motion produced a complex movement of the visual scene which traced a multi-axial path resembling an algebraic figure eight at a velocity of $36 \mathrm{deg} / \mathrm{sec}$. The roll stimulus rotated in a counterclockwise direction about the line of sight and the pitch ramp stimulus rotated from lower to upper visual fields about an axis going through the subjects' ears. Subjects were instructed to orient the hand-held wand in the vertical direction throughout each $140 \mathrm{sec}$ trial.

\section{Statistical analysis}

Root mean square (RMS) values were calculated across the period of the trial for orientation of the wand and head in the plane of interest (i.e., roll and/or pitch) and for side-to-side and anteriorposterior (a-p) center of pressure (COP). Onset latency of change in wand orientation was the time between onset of visual scene motion and the time at which wand tilt angle exceeded seven times its standard deviation during natural scene motion. An Anderson-Darling normality test at the 95\% confidence interval revealed an asymmetric, nonnormal distribution in side-to-side COP with complex visual motion $(p<0.005)$. Four of the subjects exhibited significantly different COP behavior between complex and natural visual stimuli; the remaining eight subjects did not. Thus these four subjects were treated as a separate group in this analysis. With pitch motion of the visual scene, one subject had a disproportionately high change in a-p COP $(p<0.005)$ and this subject was not included in further analyses of pitch. Paired $t$-tests were performed on the RMS values of the head, wand, and COP responses.

\section{RESULTS}

During roll, RMS values of wand orientation (ranging from $1.17^{\circ}$ to $34.81^{\circ}$ ) significantly differed from natural visual feedback $(t(11)=3.97, p<$ 0.003), but response behaviors varied across subjects. Although $75 \%$ of the subjects tilted the wand in the direction of roll, two subjects tilted in that direction continuously; three subjects tilted in the opposite direction before tilting in the direction of scene motion (mean latency of $62.77 \pm 21.32 \mathrm{sec}$ ); and the rest of the subjects fluctuated between the true and their perceived vertical (Fig. 1). RMS values of side-to-side COP shifted significantly $(t(11)=$ $4.80, p<0.003)$ in the roll direction compared to 

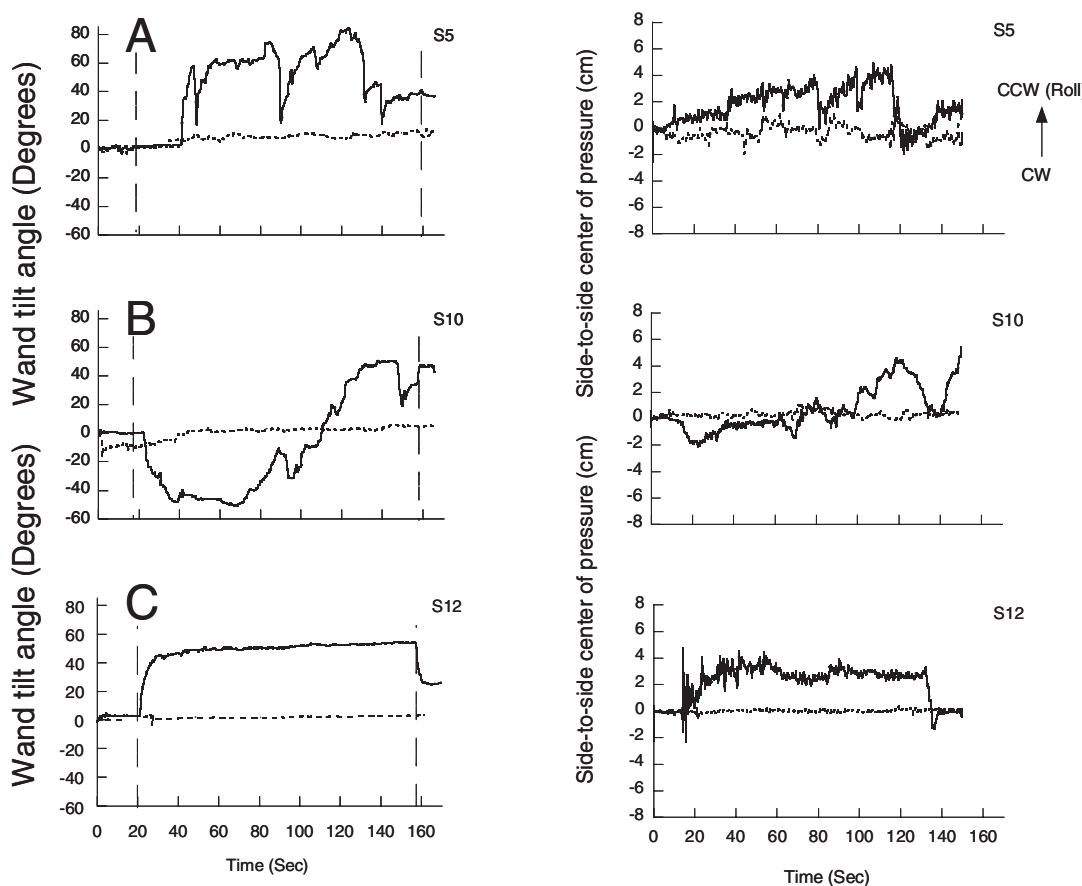

FIG. 1. (Left) Orientation of the wand during roll motion of the visual scene (bold line) and natural visual feedback (broken line) in three subjects demonstrates constant response (A), bi-directional response (B), or fluctuating (C). Dashed vertical lines mark the start and end of the scene motion. (Right) Side-to-side COP responses of the same 3 subjects shown during roll motion of the visual scene (bold line) and with natural visual feedback (broken line).

natural visual feedback (Fig. 1). RMS values of head orientation (ranging $1.38^{\circ}$ to $14.36^{\circ}$ ) were also significantly different between roll and natural visual feedback $(t(11)=4.08, p<0.003)$. Actual angles of the head and wand did not demonstrate a consistent relationship across subjects, however.

RMS values of wand orientation in pitch (ranging from $0.54^{\circ}$ to $10.87^{\circ}$ ) significantly differed from natural visual feedback $(t(10)=2.68, p<0.03)$. There was also a significant tilt of the head (ranging from $1.18^{\circ}$ to $17.26^{\circ}$ ) along the pitch axis $(t(10)=$ $2.39, p<0.04)$. RMS values of a-p COP shifted significantly $(t(10)=2.39, p<0.04)$ in pitch in $50 \%$ of the subjects and appeared as an oscillation of the body along the pitch axis or a sustained tilt either in or opposite to the direction of the pitch stimulus. When pitch and roll motions of the visual scene were combined as a complex stimulus, a significant change in side-to-side COP $(t(3)=3.72, p<0.04)$ appeared in four of the subjects when compared to responses with natural visual feedback (Fig. 2).

\section{DISCUSSION}

Postural kinematics were differentially influenced by vection depending upon the plane of mo- tion and complexity of the visual field. Despite the somatosensory inputs at each joint that could contribute to the final response, it would appear that vection from visual field motion in roll controlled whole body postural response behaviors. In pitch,

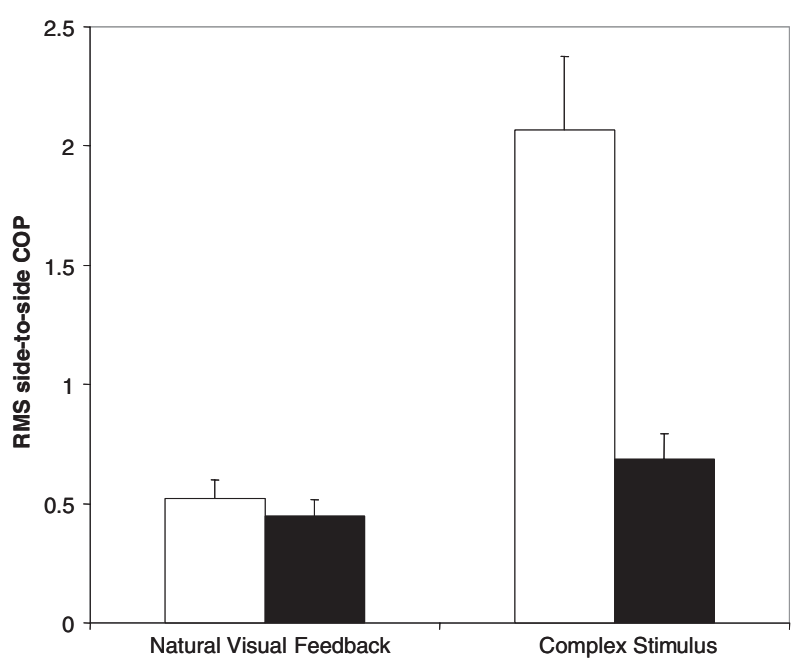

FIG. 2. RMS values of side-to-side COP in four subjects that exhibited a significant change between natural and complex visual motion (unfilled bar) and in the eight subjects that did not exhibit significance (filled bar). 
however, responses were more variable suggesting that the rich segmental inputs along the vertebral column ${ }^{7}$ or biomechanical limitations of the postural system ${ }^{6,8}$ contributed more robustly to postural perception in this plane. With complex visual motion, the impact of vection was even less apparent in the postural behavior and only a small subset of subjects exhibited a selective response to the roll component of the visual motion. This could indicate greater visual sensitivity on the part of some subjects to roll motion. It is also possible that the CNS assigned a lesser weight to visual information that does not have a real life parallel. (Note that subjects tilting up in pitch viewed the top of the CAVE where there was no scene projection; if direction of the scene were reversed, they would have viewed the floor projection and their responses may have been different.)

Thus, postural behaviors generated by the perception of self-motion appear to be a result of cortical interaction of visual and vestibular signals as well as input from other somatosensory signals. This probable on-line monitoring of spatial orientation has implications for rehabilitation interventions. Recovery of balance following a slip or trip may rely greatly on the ability to match continuously changing sensory feedback to an initial model of vertical that could be highly dependent on the visual environment and the mechanical arrangement during that particular task. It cannot be assumed that a patient, particularly one with a sensory deficit, who appears to be vertically oriented at the initiation of motion, will be able to sustain knowledge of that orientation as a task progresses.

\section{ACKNOWLEDGMENTS}

This work was supported by NIH-NIDCD (grant DC05235) and NIH-NIA (grant AG16359). CAVE ${ }^{\circledR}$ is a registered trademark of the Board of Trustees, University of Illinois.

\section{REFERENCES}

1. Dichgans, J., Held, R., Young, L.R., et al. (1972). Moving visual scenes influence the apparent direction of gravity. Science 178:1217-1219.

2. Thurrell, A.E., \& Bronstein, A.M. (2002). Vection increases the magnitude and accuracy of visually evoked postural responses. Experimental Brain Research 147:558-560.

3. Dichgans, J., \& Brandt, T. (1978). Visual-vestibular interaction: effects on self-motion perception and postural control. In: Held, R., Leibowitz, H.W., \& Teuber, H.L. (eds.), Handbook of sensory physiology. New York: Springer, pp. 755-804.

4. Fushiki, H., Takata, S., \& Watanabe, Y. (2000). Influence of fixation on circular vection. Journal of Vestibular Research 10:151-155.

5. Cruz-Neira, C., Sandin, D.J., DeFanti, T.A., et al. (1992). The CAVE automatic virtual environment. Communications of the ACM 38:64-72.

6. Keshner, E.A., \& Kenyon, R.V. (2000). The influence of an immersive virtual environment on the segmental organization of postural stabilizing responses. Journal of Vestibular Research 10:201-219.

7. Keshner, E.A. (2003). Head-trunk coordination during linear anterior-posterior translations. Journal of Neurophysiology 89:1891-1901.

8. Reymond, G., Droulez, J., \& Kemeny, A. (2002). Visuovestibular perception of self-motion modeled as a dynamic optimization process. Biological Cybernetics 87:301-314.

Address reprint requests to: Dr. E. Keshner

Rehabilitation Institute of Chicago Rm. 1406, 345 E. Superior St. Chicago, IL 60611

E-mail: eak@northwestern.edu 